\title{
Competitive exclusion in a vector-host model for the dengue fever.
}

Proposed running head: Competitive exclusion in dengue.

Zhilan Feng

Biometrics Unit

Cornell University, USA.

Jorge X. Velasco-Hernández ${ }^{1}$

Departamento de Matemáticas

UAM-Iztapalapa, México.

Keywords: Mathematical epidemiology, dengue, differential equations, vectorborne diseases, population dynamics

Please send all correspondence to the second author to the address:

Biometrics Unit

Cornell University

Ithaca NY 14853-7801

Phone: (607)-255-5488

Fax: (607)-255-4698

E-mail: jxv1@cornell.edu

${ }^{1}$ Present address: Biometrics Unit, Cornell University. Ithaca, .NY 14853-7801 


\title{
Competitive exclusion in a vector-host model for the dengue fever
}

\author{
Zhilan Feng * Jorge X. Velasco-Hernandez ${ }^{\dagger}$
}

\begin{abstract}
In this work we study a system of differential equations that models the population dynamics of an $S I R$ vector transmitted disease with two pathogen strains. This model arose from our study of the population dynamics of dengue fever. The dengue virus presents four serotypes each of which induces host immunity but only certain degree of crossimmunity to the other different serotypes. The model studied here has been constructed as a paradigm for the study of the epidemiological trends in the disease and for the theoretical study of conditions that permit coexistence in competing strains. Dengue is mainly an epidemic disease in the Americas and this model is geared to generalize this type of dynamics. In the model two different strains of virus are considered with temporary cross-immunity. The model shows the existence of an unstable endemic state ('saddle' point). The nature of this equilibrium produces a transient behavior characterized as a quasi-steady state of long duration during which both dengue serotypes co-circulate. Conditions for asymptotic stability of the equilibria are discussed together with numerical simulations. We argue that the existence of competitive exclusion in this system is due to the interplay between the host superinfection process and the frequency-dependent (vector to host) contact rates.
\end{abstract}

\section{Introduction}

Dengue fever is a disease endemic in many areas of the world that is invading and recolonizing regions where either it was absent or it had been

\footnotetext{
${ }^{*}$ Biometrics Unit, Cornell University. Ithaca NY 14853

†Departamento de Matemáticas, UAM-Iztapalapa. Apdo. Postal 55-534, Mléxico, D.F. 09340 MEXICO
} 
eradicated. Dengue is a viral disease whose etiologic agent presents up to 4 different serotypes. We construct and analyze a mathematical model about its transmission dynamics. The model is a system of differential equations that incorporates variable population size in both host and mosquito populations, two co-circulating strains and frequency dependent biting rates, all of them characteristic of the dengue virus and its transmission by mosquitoes to human hosts. The model also is designed as a theoretical framework for the discussion of some questions that arise naturally from the epidemiology of the infection, regarding the coexistence or competitive exclusion of closely related pathogen strains.

In the next section we give a basic background on the disease summarizing its epidemiological importance as well as the main features incorporated into our model. Then we proceed with the model formulation where we also discuss previous work on the principle of competitive exclusion. This section is followed by the model analysis in the case of no disease induced mortality (negligible virulence). In section 4 we discuss the computational results obtained when virulence can not be neglected and, finally, in the last section, we compare our findings with other published models that deal with superinfection, variable population size and frequency dependent infection rates.

\subsection{Dengue fever}

We start first with a brief summary of the epidemiology of dengue.

In this section we follow references [23], [22] as well as others that are indicated where appropriate.

In developing countries population growth is an important factor that contributes to the increase in the incidence of communicable diseases which affect mainly the urban poor, with infants and children among the groups particularly at risk [23], [22]. Urbanization and population growth increase the demand on basic essential services such as housing, water supply; etc., and at the same time induce conditions that increase the transmission potential of some vector borne diseases [23]. In particular, inadequacies in water supplies require large-scale water storage which are ideal breeding habitats for Aedes spp mosquitoes, the vectors of dengue ferer, dengue hemorrhagic fever and dengue shock syndrome as well as yellow fever. Changes in food habits created by the migration of rural populations to the cities lead to increase use of tinned food and more use of disposable containers that provide more breeding sites to vectors of this type. In summary population growth, 
urbanization and poverty enhance presence and transmission of infectious diseases.

Unfortunately not only dengue has increased its incidence in urban centers of the developing world but also yellow fever, malaria and Chagas disease have been benefited. The destruction of city water supplies, temporary housing for refugees, high fertility and rural to urban migration and the steady deterioration of urban environments have led to sustained growth in density and area occupied by Aedes aegypti and Aedes albopictus, two of the main vectors of dengue virus.

Other important problems of the dengue virus in the Americas and elsewhere are the public health consequences of global warming [25]. In particular, of concern is the potential spread of dengue through the vector Aedes albopictus, recently introduced to the American continent [24].

Dengue has been known for long time as occurring in tropical regions of the world. Dengue causes a spectrum of illnesses in humans ranging from clinically inapparent to severe and fatal hemorrhagic disease [9]. Classical dengue fever is generally observed in older children and adults and is characterized by sudden onset of fever, frontal headache, nausea, vomiting and other symptoms. The acute illness last for 3 to 7 days is usually benign. The hemorrhagic form of dengue and its associated dengue shock syndrome (DHF-DSS) is most commonly observed in children under the age of 15 years but it can also occur in adults [9]. It is characterized by acute onset of fever and a variety of symptoms that last 2 to 7 dars. This form of dengue can terminate in death of the patient.

Dengue is produced by viruses of the genus Togaviridae, subgenus Flavivirus. Four distinct dengue viruses have been distinguished, denoted by types $1,2,3$ and 4 . Dengue viruses can infect only a restricted number of vertebrates but is an essentially human disease [11]. Infection by any dengue virus strain produces long lasting immunity but only temporary cross immunity to other serotypes. Three of the vectors are Aedes aegypti Linnaeus, Aedes albopictus Skuse, and Aedes scutellaris Walk. Aedes aegypti mosquitoes acquire infection from infected individuals 6 to $18 \mathrm{~h}$ before onset of fever and then for the duration of the fever. A minimum extrinsic incubation period of 8 to 14 days is required after an infective blood meal before the mosquito becomes infectious. The infection in the vector is for life. Dengue virus is transmitted in two cycles: urban and sylvan although, as mentioned before, it is predominantly a human virus[11].

There are no effective programs for vector control and, as a consequence, the absolute numbers of dengue infection and dengue infection rates have 
increased during the last 40 years [12]. Unfortunately, countries where positive results exist for vector eradication have been suffering from epidemic outbreaks: the disease is coming back. Dengue viruses where introduced in the Americas around 1960 and since, dengue has been reported in countries where it was absent before as Cuba, México [19], the United States, most Central America, Ecuador, Perú, Paraguay, Bolivia, Argentina and Brazil [12].

Dengue transmission occurs throughout the year in endemic tropical areas but there exists, however, a distinct cyclical pattern associated with the rainy season [9]. In particular, in Thailand where the vector life cycle is highly domiciliary, temperature and humidity conditions during the rainy season favor survival of infected mosquitoes. In the Americas the situation is different since in these areas larvae develop in the outdoors. Here, peak transmission takes place in the days of highest rainfall and warmer temperatures season [9].

We can distinguish two types of dengue: classical dengue which is essentially benign in the long run and DHF-DSS that can cause death. In regions where mosquito and humans exists, an introduction of dengue virus may produce an epidemic depending upon a) the strain of the rirus (influencing magnitude and duration of viremia), b) the susceptibility of the human population, c) the density, behavior, and competence of the mosquito vector population, and d) the introduction of the virus into an area where it has contact with the local mosquito population [9]. Severity of dengue fever has been associated with secondary dengue infections although its causes are far from being explained. Epidemiological studies in Thailand suggest that an important risk factor for DHF-DSS is the presence of preexisting dengue antibody at subneutralizing levels. Also, endemic DHF-DSS is found in areas where Aedes aegypti densities are high and dengue virus of multiple types are endemic. Moreover, DHF-DSS is associated with individuals older than 1 year with a secondary type antibody response and with primary infections in newborn babies whose mother where immune to dengue [9], [11]. These facts led to the formulation of the secondary infection or immune enhancement hypothesis to explain it [9]. This hypothesis states that only those persons experiencing a second infection with heterologous dengue serotype present DHF-DSS. In particular it has been found that only secondary dengue-2 disease is immunologically enhanced and that infection with this virus serotype cause the majority of DSS cases [12]. Other factors are also associated with DHF-DSS. These are sex (more frequent infections in females), nutritional status (higher incidence in well-nourished babies of 
middle and upper socioeconomic class), and the interval between first and second infections.

To conclude this brief review of the epidemiology of dengue: we show in Table 1 a summary of dengue epidemic in sereral countries of the world.

\section{Superinfection and coexistence}

As seen in the previous section, in dengue certain sequences of infection appear to be more damaging to the host than others. We decided to model this process through the use of a susceptibiiity coefficient similarly to the one used by [13] and [4]. This coefficient allows us to explore varying degrees of susceptibility to secondary infections and their effect on the asymptotic dynamics of the disease. Through the analysis of the model we attempt to explore the consequences of the coupling of two populations that differ in the infection pathway that the virus follows ( $S I R$ with superinfection in the host, $S I$ in the vector), the effect of the frequency-dependent infection rate in the coupling of both systems, and to compare it with published results dealing with the evolution of virulence in directly transmitted diseases.

Most diseases are produced by an spectrum of closely related pathogens rather than by single strains and dengue is c'early an example of this assertion. In fact, in this disease an analogous phenomenon to superinfection, as defined by Nowak and May [21] and May and Nowak [16] takes place. One strain invades the host population. produces a brief period of temporary immunity to other strains but when this immunity is lost it leares the host susceptible to reinfection with another strain. The particular process that takes place in dengue fever is that before reinfection can occur, there is a period where the host is resistant, in varying degrees, to all strains, not only the one that produced the primary infection [9]. One important theoretical problem that can be discussed when superinfection occurs is that of the coexistence of all strains or the erentual ext:nction of some of them. This problem has been theoretically exp:ored by several authors [13]. [2], [6], [5].

In dengue fever we are confronted witt a vector-transmitted disease, co-circulating strains, certain degree of cross-immunity or even increased susceptibility to infection, and a variable hos: population size. Thus we can address certain issues on the evolution of vilence. Nowak and May [21] and Tilman [27] have explored the irterrelation that exists between virulence and superinfection in a constant host population. It is expected that conditions for coexistence of different strains dejend strongly on the constant 
population assumption.

There are numerous published results discussing the problem of coexistence in pathogen-host interactions. Levin and Pimentel [13]constructed a mathematical $S I$ model where the population in the absence of disease would grow exponentially. Two strains with different virulences compete with each other. The most virulent strain can takeover hosts already infected with the less virulent strain. Under these assumptions a globally stable equilibrium is possible where both strains may coexist [3]. The stability of the positive equilibrium is only guaranteed for certain range of values of superinfection. Outside this range one of the boundary equilibria is asymptotically stable. Bremermann and Thieme [2] postulate a competitive exclusion principle in a mathematical model where several strains compete for a single host population. The pathogens differ on their virulence. In this model the assumption that virulence is a strictly convex function of the transmission rate implies that the evolution of virulence leads to a transmission rate that maximizes the basic reproductive number of the pathogen [2]. This model describes a $S I R$ epidemic in a population with variable size. Castillo-Chavez et al [6] find, for a $S I S$ two-sex model with variable population size, that competitive exclusion is the norm: the strain with the highest reproductive number persists in both host types. Mena-Lorca, Velasco-Hernandez and Castillo-Chavez [17] have studied the effect of variable population, virulence and density-dependent population regulation. The interplay between this demographic process and the presence of a potentially fatal and conditions for coexistence and competitive exclusion of the two pathogen strains were established. In this model too, coexistence is feasible only in certain window of parameter values. Previous models for dengue fever are those reported in [26] and [20]. The first one is a cost-effectiveness model for the management of dengue. It thus addresses different issues than the ones we are concerned with in this paper. The second model follows the same basic methodology that we adopt here. The model in [20] explicitly establishes the existence of an incubation or latent period where both mosquitoes and humans have been exposed to a single virus strain but are not yet infectious. Both total populations of hosts and vectors are considered constant.

In the model that we analyze here, we consider variable population sizes of both hosts and vector populations, we do not incorporate the exposed compartment, but include instead the existence of a second co-circulating strain that can produce secondary infections in those individuals either susceptible or already recovered from a primary infection with a different strain. 


\subsection{Model equations}

Consider a human population settled in a region where a mosquito population of the genus Aedes is present and carrier of the dengue virus.

Model equations then stand as follows $\left({ }^{\prime}=d / d t\right)$ :

$$
\begin{aligned}
S^{\prime}(t) & =h-\left(B_{1}+B_{2}\right) S-u S \\
I_{1}^{\prime}(t) & =B_{1} S-\sigma_{2} B_{2} I_{1}-u I_{1} \\
I_{2}^{\prime}(t) & =B_{2} S-\sigma_{1} B_{1} I_{2}-u I_{2}, \\
Y_{1}^{\prime}(t) & =\sigma_{1} B_{1} I_{2}-\left(e_{1}+u+r\right) Y_{1} \\
Y_{2}^{\prime}(t) & =\sigma_{2} B_{2} I_{1}-\left(e_{2}+u+r\right) Y_{2} \\
R^{\prime}(t) & =r\left(Y_{1}+Y_{2}\right)-u R
\end{aligned}
$$

and

$$
\begin{aligned}
M^{\prime}(t) & =q-\left(A_{1}+A_{2}\right) M-\delta M \\
V_{1}^{\prime}(t) & =A_{1} M-\delta V_{1} \\
V_{2}^{\prime}(t) & =A_{2} M-\delta V_{2}
\end{aligned}
$$

In the above $N=S+I_{1}+I_{2}+Y_{1}+Y_{2}+R$ and $T=M+V_{1}+V_{2}$ are the host and vector total population sizes respectively (see Table 2 for other parameter definitions and values). Primary infections in human hosts are produced by either of the two strains at rates

$$
B_{i}=\beta_{i} V_{i} /\left(c+\omega_{h} N\right)
$$

for $i=1,2$ (in vector to host transmission). Primary infections in vectors are produced at rates

$$
A_{i}=\alpha_{i}\left(I_{i}+Y_{i}\right) /\left(c+\omega_{v} N\right) .
$$

These function forms are used since we are modeling frequency-dependent disease transmission. Both are special cases of the Holling type II functional response [7]. Moreover, these expressions are also generalizations of the basic formulation in the Ross-Macdonald model for Malaria transmission as discussed in [1] and for Chagas disease in [28]. The assumptions reflect the fact that in a vector-host interaction, mosquitoes are able to choose their food source in a frequency dependent fashion while hosts cannot.

We assume that once a mosquito is infected it never recovers and, moreover, it can not be reinfected with a different strain of virus. 
Secondary infections, therefore, may take place only in the host. We assume that these occur by contact with infected mosquitoes with a different strain. Two cases develop: either previously $I_{1}$ individuals are infected by strain 2, through contact with infected mosquitoes $V_{2}$, becoming $Y_{2}$ hosts, or previously $I_{2}$ individuals are infected with strain 1 , through contact with $V_{1}$ mosquitoes, to become $Y_{1}$ infected hosts, at rates $\sigma_{1} B_{1} I_{2}$ and $\sigma_{2} B_{2} I_{1}$, respectively. Here, $\sigma_{i}$ is a positive real number that may mimic the immune enhancement hypothesis in dengue infections discussed in the introduction. We consider this type of dynamics an special case of superinfection as discussed in [21]. In dengue, however, the immunity developed after infection is a factor that does not appear in the models developed by the cited authors. Moreover, in the dengue case the cross-immunity is a two-way factor (either of the primary infected populations can be reinfected with the other strain) that appears whenever a primary infection takes place, regardless of the strain. In the case $\sigma<1$, we have a certain degree of cross-immunity which diminishes the infection rate of the secondary strain. General results on the effects of cross-immunity [21], [17] in SIS and SI models respectively, indicate that for certain values of $\sigma$ coexistence of competing strains is possible. As will be seen later, the existence of a two-way cross-immunity together with the induction of permanent immunity specific to each indiridual strain, and the fact that the contact rates from vector to host are frequency dependent, prevent coexistence. The generic outcome of our model is competitive exclusion although in some cases in a very long time scale.

Thus, in this model, if $\sigma_{i}<1$, primary infections confer partial immunity to strain $i$; if $\sigma_{i}=1$ secondary infections with strain $i$ take place as if they were primary infections, and if $\sigma_{i}>1$ primary infections increase susceptibility to strain $i$. Once an individual has suffered from both infections it gets immunity to both strains at a rate $r$ independent of the sequence of infections.

Since the equation for the total vector population is

$$
T^{\prime}=q-\delta T,
$$

we have that as $t \rightarrow \infty, T(t) \rightarrow q / \delta$. This allows us to substitute $M=$ $q / \delta-V_{1}-V_{2}$ making the equation for $M$ in (2) redundant. 


\section{Model analysis}

Let

$$
\begin{gathered}
\Omega=\left\{\left(S, I_{1}, I_{2}, Y_{1}, Y_{2}, R, V_{1}, V_{2}\right): S+I_{1}+I_{2}+Y_{1}+Y_{2}+R \leq h / u,\right. \\
\left.V_{1}+V_{2} \leq q / \delta\right\}
\end{gathered}
$$

be the set bounded by the total host and vector population in the absence of disease. Note that we can immediately identify three equilibrium solutions to (1-2). The disease-free equilibrium $E_{0}^{*}=\left(S^{*}, 0,0,0,0,0,0,0\right)$ and the other two (boundary) equilibria

$$
E_{1}^{*}=\left(S_{1}^{*}, I_{1}^{*}, 0,0,0,0, V_{1}^{*}, 0\right),
$$

where only strain 1 survives, and

$$
E_{2}^{*}=\left(S_{2}^{*}, 0, I_{2}^{*}, 0,0,0,0, V_{2}^{*}\right)
$$

where only strain 2 survives.

\subsection{Basic reproductive number}

In a situation when co-circulating strains are present in a vector-host system, the colonization or invasion of susceptible hosts is a two-sided erent. Each pathogen strain has to manage to be transmitted from an infected type 1 host to a susceptible type 2 host, and back again from a now infected type 2 host to a susceptible type 1 host. The invasion criterion is summarized in the basic reproduction number. This is defined as the number of secondary infection that an infectious individuals produces in a population where all hosts are susceptible.

To find the basic reproductive number of our model we equate (1-2) to zero and solve each one for $S, I_{i}, Y_{i}, R, V_{i}$ in terms of $B_{i}$ and $A_{i}$ to obtain

$$
\begin{aligned}
S & =h /\left(u+B_{1}+B_{2}\right), \\
I_{i} & =B_{i} S /\left(u+\sigma_{j} B_{j}\right), i \neq j, i, j=1,2, \\
Y_{i} & =\sigma_{i} B_{i} I_{j} /\left(u+e_{j}+r\right), i \neq j, i, j=1,2, \\
R & =r\left(Y_{1}+Y_{2}\right) / u \\
V_{1} & =q A_{1} /\left(\delta+A_{1}+A_{2}\right), \\
V_{2} & =q A_{2} /\left(\delta+A_{1}+A_{2}\right) .
\end{aligned}
$$

Let $K=\left(B_{1}, B_{2}, A_{1}, A_{2}\right)^{\prime}$ ( $/$ denotes transpose) be a four dimensional vector. Substituting the above expressions into the definition of $B_{i}$ and $A_{i}$ we get 
a system of four non-linear algebraic equations in terms of $B_{i}$ and $A_{i}$. We denote this system by $\Phi(K)$. The fixed points of $\Phi(K)$ give, by construction, all the equilibrium points of (1-2).

This change of variables allows an easy computation of the next generation operator [8] and the associated basic reproduction number. By construction, the next-generation operator is simply the Jacobian of $\Phi$ evaluated at the disease-free equilibrium (given by $B_{1}=B_{2}=A_{1}=A_{2}=0$ ):

$$
D \Phi(0)=\left(\begin{array}{cccc}
0 & 0 & \frac{\beta_{1} h}{u^{2}\left(c+h \omega_{h} / u\right)} & 0 \\
0 & 0 & 0 & \frac{\beta_{2} h}{u^{2}\left(c+h \omega_{h} / u\right)} \\
\frac{\alpha_{1} q}{\delta^{2}\left(c+h \omega_{v} / u\right)} & 0 & 0 & 0 \\
0 & \frac{\alpha_{2} q}{\delta^{2}\left(c+h \omega_{v} / u\right)} & 0 & 0
\end{array}\right) .
$$

The basic reproduction number is therefore

$$
\mathcal{R}_{0}=\max \left\{\sqrt{R_{1}}, \sqrt{R_{2}}\right\}
$$

with

$$
R_{i}=\frac{\alpha_{i} \beta_{i} h q / \delta u}{u \delta\left(c+h \omega_{h} / u\right)\left(c+h \omega_{v} / u\right)} .
$$

Note that the basic reproductive number is a generalization of the RossMacdonald basic reproductive number for malaria. We want to remark that these expressions arose from a vector-transmitted disease model with variable population size, and contact rates of Holling type II to account for frequency-dependence in the contact process. Therefore, our equations are a correct generalization of the Ross-Macdonald model for multiple strains and variable population size in both host and vector.

It follows then that if $\mathcal{R}_{0}>1$, then the disease is able to invade the host population. Otherwise, if $\mathcal{R}_{0} \leq 1$ the virus eventually disappears from the host population (local result).

\subsection{Boundary equilibria}

We are interested in the conditions that guarantee that at least one of the viral strains remains in the population. To this end we proceed now to analyze the so-called boundary (non-trivial) equilibria of our model. These points correspond to steady-states where only one of the strains persists in the population, the other being driven to extinction through competition. The existence of the boundary equilibria is determined by the relative magnitudes of the basic reproductive numbers of each strain. 
Whenever $R_{j}<1$ then $B_{j}, A_{j}$ both vanish. Therefore, $R_{i}>1, R_{j}<$ $1, i \neq j, i, j=1,2$, implies the existence of $E_{i}^{*}$ which is also a unique endemic equilibrium (but where only one strain is present). Under these conditions the asymptotic dynamics of system (1-2) is completely contained in an invariant manifold $\Omega_{i} \subset \Omega$ defined as

$$
\Omega_{i}=\left\{\left(S_{i}, I_{i}, V_{i}\right): 0<S_{i}+I_{i} \leq h / u, 0<V_{i} \leq q / \delta\right\} .
$$

Taking $V_{j}=I_{j}=Y_{j}=R=0$ we find the dynamics of our model within $\Omega_{i}$ is described by the equations

$$
\begin{aligned}
S^{\prime}(t) & =h-B_{i} S-u S, \\
I_{i}^{\prime}(t) & =B_{i} S-u I_{i}, \\
V_{i}^{\prime}(t) & =\frac{\alpha_{i} I_{i}}{c+\omega_{i} N}\left(q / \delta-V_{i}\right)-\delta V_{i},
\end{aligned}
$$

with the total host population size (in $\Omega_{i}$ ) satisfying the equation

$$
N^{\prime}(t)=h-u N .
$$

In (3) the unique (non-trivial) equilibrium point is the projection of $E_{i}^{*}$ onto the subspace $\Omega_{i}$. Moreover, virulence (extra mortality induced by the virus) plays no role in this asymptotic system.

Since $N(t) \rightarrow h / u$ as $t \rightarrow \infty$, (3) can be reduced further to the equations

$$
\begin{aligned}
I_{i}^{\prime}(t) & =\frac{\beta_{i} V_{i}}{c+\omega_{h} h / u}\left(h / u-I_{i}\right)-u I_{i} \\
V_{i}^{\prime}(t) & =\frac{\alpha_{i} I_{i}}{c+\omega_{i} h / u}\left(q / \delta-V_{i}\right)-\delta V_{i}
\end{aligned}
$$

which is the same system as the one studied for Malaria by Aron and May [1]. Therefore, whenever $R_{i}>1$, this limiting system is globally asymptotically stable in $\Omega_{i}[18]$.

\subsubsection{Boundary equilibria and virulence}

Before proceeding to the derivation of threshold parameters for the existence and stability of the boundary equilibria, we comment on the general properties to be expected when the virulences $e_{i}$ are positive quantities. 
The local stability analysis of $E_{i}^{*}$ (equilibrium with only strain $i$ present) can be looked at by computing $D \Phi\left(E_{i}^{*}\right)$. The eigenvalues of this matrix around this equilibrium are

$$
\lambda_{i}=R_{i}^{-1}, \text { and } \mu_{i}=R_{j} \psi\left(\sigma_{i}, \sigma_{j}, R_{i}\right), i \neq j,
$$

where $\psi$ is a multiplicative perturbation of $R_{j}$ that depends on the basic reproductive number of strain $i$ as well as on the superinfection indices $\sigma_{1}$ and $\sigma_{2}$. This perturbation has the general form

$$
\psi=\psi_{i}\left(\sigma_{i}, R_{i}\right)+\psi_{j}\left(\sigma_{j}, R_{i}\right), i \neq j .
$$

where

$$
\psi_{i}=\frac{c_{1}}{u+c_{2} \sigma_{i}\left(R_{i}-1\right)}, \psi_{j}=\frac{c_{3} \sigma_{j}\left(R_{i}-1\right)}{c_{4}\left(e_{j}+r+u\right)},
$$

with $c_{1}, c_{2}, c_{3}$ and $c_{4}$ positive constants. In particular $v$ satisfies $\partial \psi_{i} / \partial \sigma_{i}<$ 0, and $\partial \psi_{j} / \partial \sigma_{j}>0$ and $\partial \psi_{j} / \partial e_{j}<0$.

Note that the stability of the equilibrium point $E_{i}^{*}$ is favored $\left(\mu_{i}\right.$ tends to reduce its magnitude) when the virulence (extra host mortality induced by the virus) of strain $j$ is large.

Moreover, in the limiting case were $\sigma_{i}=\sigma_{j}=0$ (no superinfection occurring), $\psi=R_{1}^{-1}$. Therefore, the condition $R_{j}<1<R_{i}$ makes $\Phi$ a local contraction around $E_{i}^{*}$. Thus, the local asymptotic stability of $E_{1}^{*}$ in $\Omega$ follows.

However, if $0<\sigma_{i}<\sigma_{j}, E_{i}^{*}$ may cease to be an attractor under $\Phi$ (with the condition $R_{j}<1<R_{i}$ holding). In this case $E_{i}^{*}$ is still a global attractor in $\Omega_{i}$ and a saddle point in $\Omega$.

It is important to mention that whenever $R_{i}>1$ and $R_{j}>1$ simultaneously, both equilibria $E_{i}^{*}$ and $E_{j}^{*}$ exists. Their stability properties depend on the magnitude of $\mu_{i}$ and $\mu_{j}$ respectively ( $\lambda_{i}$ and $\lambda_{j}$ are alwars less than one whenever $R_{i}>1$ and $R_{j}>1$ ). In the following section we provide more detailed conditions for the existence and stability properties of the boundary equilibrium for $\sigma_{i}$ positive and $e_{i}=0$ (for $e_{i}>0$ numerical simulations give the same result).

\subsection{Threshold parameters when virulence is negligible}

To obtain precise results on the existence and stability properties of equilibrium points of our model we neglect mortality due to disease. This assumption is not justifiable in all regions where dengue is endemic but it is a first approximation to the analysis of our model. 
In the absence of virulence, the host population is asymptotically constant and therefore we can reduce the dimension of our model equations by one. We drop the equation for $S$. Let $\omega_{h}=\omega_{v}=\omega, \hat{N}=h / u$ and $\hat{T}=q / \delta$ and take $e_{i}=0$.

To facilitate the analysis and interpretation of our results we compute the Jacobian matrix of (1-2) by reordering it as indicated in (4) below.

Consider the boundary equilibrium

$$
E_{1}^{*}=\left(V_{1}^{*}, I_{1}^{*}, V_{2}^{*}, I_{2}^{*}, Y_{1}^{*}, Y_{2}^{*}, R^{*}\right)
$$

where

$$
V_{2}^{*}=I_{2}^{*}=Y_{1}^{*}=Y_{2}^{*}=R^{*}=0
$$

and

$$
V_{1}^{*}=\frac{u \delta\left(R_{1}-1\right)}{b_{1}\left(\delta+a_{1} \hat{N}\right)}, I_{1}^{*}=\frac{u \delta\left(R_{1}-1\right)}{a_{1}\left(b_{1} \hat{T}+u\right)}
$$

with

$$
a_{i}=\frac{\alpha_{i}}{c+\omega \hat{N}}, b_{i}=\frac{\beta_{i}}{c+\omega \hat{N}} .
$$

With this new order the Jacobian has the form

$$
J\left(E_{1}^{*}\right)=\left(\begin{array}{cc}
G_{1} & G_{2} \\
0 & G_{4}
\end{array}\right),
$$

where

$$
\begin{gathered}
G_{1}=\left(\begin{array}{cccc}
-\delta-a_{1} I_{1}^{*} & a_{1}\left(\hat{T}-V_{1}^{*}\right) \\
b_{1}\left(\hat{N}-I_{1}^{*}\right) & -u-b_{1} V_{1}^{*}
\end{array}\right), \\
G_{4}=\left(\begin{array}{ccccc}
-\delta & a_{2}\left(\hat{T}-V_{1}^{*}\right) & 0 & a_{2}\left(\hat{T}-V_{1}^{*}\right) & 0 \\
b_{2}\left(\hat{N}-I_{1}^{*}\right) & -u-\sigma_{1} b_{1} V_{1}^{*} & 0 & 0 & 0 \\
0 & \sigma_{1} b_{1} V_{1}^{*} & -(u+r) & 0 & 0 \\
\sigma_{2} b_{2} I_{1}^{*} & 0 & 0 & -(u+r) & 0 \\
0 & 0 & r & r & -u
\end{array}\right) .
\end{gathered}
$$

The eigenvalues of $J\left(E_{1}^{*}\right)$ are given by the eigenvalues of $G_{1}$ and $G_{4}$. When $R_{1}>1, G_{1}$ has two eigenvalues with negative real parts. Eigenvalues of $G_{4}$ are given by $-u,-(u+r)$ and by the roots of the polynomial

$$
\lambda^{3}+A \lambda^{2}+B \lambda+C=0
$$


where

$$
\begin{aligned}
& A=2 u+\sigma_{1} b_{1} V_{1}^{*}+r+\delta \\
& B=(u+r)\left(u+\sigma_{1} b_{1} V_{1}^{*}\right)+\delta\left(u+\sigma_{1} b_{1} V_{1}^{*}\right)-u \delta \frac{R_{2}}{R_{1}}-\sigma_{2} a_{2} b_{2} \frac{\delta V_{1}^{*}}{a_{1}} \\
& C=\delta\left(u+\sigma_{1} b_{1} V_{1}^{*}\right)(u+r)-(u+r) u \delta \frac{R_{2}}{R_{1}}-\left(u+\sigma_{1} b_{1} V_{1}^{*}\right) \sigma_{2} a_{2} b_{2} \frac{\delta V_{1}^{*}}{a_{1}}
\end{aligned}
$$

In the above we have used the equivalencies

$$
\left(\hat{N}-I_{1}^{*}\right)\left(\hat{T}-V_{1}^{*}\right)=\frac{\hat{N} \hat{T}}{R_{1}}, \frac{u}{b_{1} V_{1}^{*}}=\frac{\delta+a_{1} \hat{N}}{\delta\left(R_{1}-1\right)}, I_{1}^{*}\left(\hat{T}-V_{1}^{*}\right)=\frac{\delta V_{1}^{*}}{a_{1}} .
$$

Our threshold parameters are given in terms of bounds for the superinfection coefficients $\sigma_{1}$ and $\sigma_{2}$. Thus, they set bound for the level of induced resistance or increased susceptibility that each strain produces in the host.

Let

$$
\begin{aligned}
\sigma_{1}^{*} & =\max \left\{0,\left(\frac{R_{2}}{R_{1}}-1\right) \frac{\delta+a_{1} \hat{N}}{\delta\left(R_{1}-1\right)}\right\} \\
\sigma_{2}^{*} & =f\left(\sigma_{1}\right)=\frac{\delta(u+r)}{a_{2} b_{2} I_{1}^{*}\left(\hat{T}-V_{1}^{*}\right)}\left(1-\frac{u}{u+\sigma_{1} b_{1} V_{1}^{*}} \frac{R_{2}}{R_{1}}\right) .
\end{aligned}
$$

Then the following hold

\section{Lemma 1:}

1. $\sigma_{1}>\sigma_{1}^{*}$ implies $\sigma_{2}^{*}>0$;

2. $\sigma_{1}>\sigma_{1}^{*}$ and $\sigma_{2}<\sigma_{2}^{*}$ implies $A>0, B>0, C>0$ and $A B>C$;

3. $\sigma_{1}<\sigma_{1}^{*}$ or $\sigma_{2}>\sigma_{2}^{*}$ imply $C<0$.

The proofs of 1 and 3 are straightforward. To show 2, let $\sigma_{1}>\sigma_{1}^{*}$ and $\sigma_{2}<\sigma_{2}^{*}$. It is easy to see that $\sigma_{2}<\sigma_{2}^{*}$ implies $C>0$. Note that

$$
\delta\left(u+\sigma_{1} b_{1} \Gamma_{!}^{-*}\right)>u \delta \frac{R_{2}}{R_{1}} .
$$

Hence

$$
B\left(u+\sigma_{1} b_{1} V_{1}^{*}\right) \geq(u+\delta)\left(u+\sigma_{1} b_{1} V_{1}^{*}\right) \varepsilon-\left(u+\sigma_{1} b_{1} V_{1}^{*}\right) \sigma-2 a_{2} b_{2} I_{1}^{*}\left(\hat{T}-V_{1}^{*}\right) \geq C,
$$


but $C>0$, therefore $B>0$. To show that $A B>C$ we note that $\sigma_{2}<f\left(\sigma_{1}\right)$, thus obtaining

$$
\sigma_{2} a_{2} b_{2} I_{1}^{*}\left(\hat{T}-V_{1}^{*}\right) \leq \delta(u+r)-u \delta \frac{u+r}{u+\sigma_{1} b_{1} V_{1}^{*}} \frac{R_{2}}{R_{1}} .
$$

It follows then that

$$
\begin{aligned}
A B \geq & \left(2 u+\sigma_{1} b_{1} V_{1}^{*}+r+\delta\right) \cdot\left((u+r)\left(u+\sigma_{1} b_{1} V_{1}^{*}+\delta\right)+\delta\left(u+\sigma_{1} b_{1} V_{1}^{*}\right)\right. \\
& \left.-u \delta \frac{R_{2}}{R_{1}}-\delta(u+r)+u \delta \frac{u+r}{u+\sigma_{1} b_{1} V_{1}^{*}} \frac{R_{2}}{R_{1}}\right) \\
> & (u+r)\left(\delta\left(u+\sigma_{1} b_{1} V_{1}^{*}\right)-u \delta \frac{R_{2}}{R_{1}}\right)
\end{aligned}
$$

Since

$$
C<\delta\left(u+\sigma_{1} b_{1} V_{1}^{*}\right)(u+r)-(u+r) u \delta \frac{R_{2}}{R_{1}}
$$

it follows that

$$
A B>C \text {. }
$$

Using the Routh-Hurwitz criteria we have the following result:

\section{Corollary}

The boundary equilibrium $E_{1}^{*}$ is locally asymptotically stable if $\sigma_{1}>\sigma_{1}^{*}$, and $\sigma_{2}<\sigma_{2}^{*}$. Otherwise it is unstable.

Lemma 1 and the corollary say that whenever the superinfection coefficient of the first strain is above threshold there are values of the second superinfection coefficient that give asymptotic stability of the boundary equilibrium $E_{1}^{*}$. Note that the value of the thresholds $\sigma_{1}^{*}$ and $\sigma_{2}^{*}$ is not specified. This means that the asymptotic stability of $E_{1}^{*}$ is guaranteed in principle either when the first strain induces resistance or when it increases susceptibility to the second strain.

Using the definition of $f$ given in (5) we have

\section{Lemma 2:}

1. If $R_{2}<R_{1}$, then $\sigma_{1}^{*}=0, f\left(\sigma_{1}^{*}\right)=f(0)>0, f^{\prime}\left(\sigma_{1}\right)>0$.

2. If $R_{2}>R_{1}$, then $\sigma_{1}^{*}>0, f\left(\sigma_{1}^{*}\right)=0, f(0)<0$ and $f^{\prime}\left(\sigma_{1}\right)>0$. 
Define $D_{1}=\lim _{\sigma_{1} \rightarrow \infty} f\left(\sigma_{1}\right)$. Then the region of stability of $E_{1}^{*}$ given by Lemma 2 is shown in Figures 1 and 2 for both cases $(f(0)>0$ and $f(0)<0)$.

Using the symmetry between the two dengue strains, we can perform a similar analysis for the other boundary equilibrium $E_{2}^{*}$ (where the second strain wins). In this case we have $V_{2}^{*}>0$ and $I_{2}^{*}>0$, and we can define

$$
\begin{aligned}
\sigma_{2}^{\bullet} & =\max \left\{0,\left(\frac{R_{1}}{R_{2}}-1\right) \frac{\delta+a_{2} \hat{\Lambda}^{-}}{\delta\left(R_{2}-1\right)}\right\} \\
\sigma_{1}^{\bullet} & =g\left(\sigma_{2}\right)=\frac{\delta(u+r)}{a_{1} b_{1} I_{2}^{*}\left(\hat{T}-V_{2}^{*}\right)}\left(1-\frac{u}{u+\sigma_{2} b_{2} V_{2}^{*}} \frac{R_{1}}{R_{2}}\right) .
\end{aligned}
$$

Without loss of generality assume $R_{1}>R_{2}>1$. Then we can draw a bifurcation diagram in parameter space $\left(\sigma_{1}, \sigma_{2}\right)$. See Figure 3.

Putting together the three lemmas and the corollary above, we summarize our results in the following lemma about the local stability properties of both boundary equilibria (where we use the definition of $g$ given in (6)):

\section{Lemma 3:}

1. $E_{1}^{*}$ is locally asymptotically stable if $\sigma_{2}<f\left(\sigma_{1}\right)$ for every $\sigma_{1}>0$, and unstable if $\sigma_{2}>f\left(\sigma_{1}\right)$.

2. $E_{2}^{*}$ is locally asymptotically stable if $\sigma_{2}>g^{-1}\left(\sigma_{1}\right)$ for erery $\sigma_{1}>0$, and unstable if $\sigma_{2}<g^{-1}\left(\sigma_{1}\right)$.

3. $E_{1}^{*}$ and $E_{2}^{*}$ are locally asymptotically stable if $g^{-1}\left(\sigma_{1}\right)<\sigma_{2}<f\left(\sigma_{1}\right)$.

Note that it is possible to have threshold ralues of $\sigma_{1}^{*}$ and $\sigma_{2}^{*}$ such that both $E_{1}^{*}$ and $E_{2}^{*}$ are locally asymptotically stable. This conclusion indicates, at the very least, that there are situations where the asymptotic dynamics of our model depends on the initial conditions.

\section{Characterization of the interior endemic equi- librium}

In this section we present the conclusions that we obtained from numerical simulations. They provide strong evidence of the existence of an interior endemic equilibrium, that is, an equilibrium point with positive densities of both infected host types. In these simulations we have explored the 
interdependence of three key parameters: the basic reproductive numbers of each strain, the superinfection coefficients and the disease-induced death rate for each strain. The dimension of the limiting equations (1) and (2) has one less dimension than the original system if the virulences for both strains are set to zero. This is the case analyzed in the previous section. However, when virulence is not negligible the populations of hosts and vectors are not constant. It is known [17] that variable population size can have a very dramatic effect on the result of a competitive interaction. In particular it can 'reduce' the area of parameter space on which coexistence is possible (compared with the equivalent model with constant total population size). The following conclusions were found through the numerical simulation of model (1-2) for the case $e_{i}>0$.

\section{Conclusions:}

1. Whenever $R_{i}>1$ for $i=1,2$ there exists an equilibrium point in the interior of $\Omega$. This point (represented in the figure by a $\triangle$ ) has a local unstable and a local stable manifold of positive dimension (Figure 5).

2. If $R_{i}>1>R_{j}$ then the boundary equilibrium $E_{j}^{*}$ and the interior endemic equilibrium do not exist and the boundary equilibrium $E_{i}^{*}$ is globally asymptotically stable (Figure 4).

3. When $R_{i}>R_{j}>1$, the superinfection coefficients $\sigma_{1}$ and $\sigma_{2}$ may change the asymptotic behavior of the system, rendering strain $j$ as the winner over strain $i$ (which would be the winner if $\sigma_{1}=\sigma_{2}=1$, see Figure 6).

4. When $R_{i}>R_{j}>1$ and both boundary equilibria are locally asymptotically stable, there exists a separatrix that cuts $\Omega$ into two disjoint basins of attraction (one for each boundary equilibrium). The location of the endemic equilibrium is indicated by a $\Delta$ in Figure 5 .

The model simulations show that there is no long term persistence of both strains in the host population. However, the unusual nature of the endemic equilibrium (a 'saddle' point) produces a somewhat prolonged (years of duration) quasi-steady state provided $R_{i}$ are both greater than one. Given the time-scale inherent to the disease (months), this quasi-steady state would look as an stable endemic equilibrium (see Figures 5 and 6). Under these conditions there are two possibilities depending on how many boundary equilibria are locally stable. If only one of them is locally asymptotically stable, 
according to our computer simulations the corresponding dengue strain will be the winner. However, although the primary infection is driven by the strain with the highest reproduction number, if this infection enhances secondary infections with the other strain, the long-term behavior of the disease can be changed (see Figure 6). The strain with the smallest reproductive number may end up persisting in the host with the consequent extinction of the other one.

The second possibility occurs when both boundary equilibria exist and both are locally asymptotically stable. In this case the outcome of the interaction depends on the initial conditions (Figure 5).

\section{Discussion}

The incorporation of full vector-host dynamics in a multiple strain epidemiological system has been partially analyzed in this work. Conditions for existence and stability properties of the interior endemic equilibrium point are somewhat unusual in this model. Although the existence of the endemic equilibrium is still a function of the magnitude of the basic reproduction numbers of each of the strains, whenever both basic reproduction numbers are greater than one, the endemic equilibrium is always unstable with stable and unstable manifolds of non-zero dimension. The existence of an interior endemic equilibrium point with this characterization imposes a potentially unpredictable outcome of the evolution of the infection in the host population. A new infection after the primary epidemic burst will settle to a transient, apparently stable, low level of endemicity where co-circulating strains might be present. However, as time passes, one of the strains will eventually and steadily increase its prevalence until most of the susceptible population is infected. The other strain will disappear.

The simulations performed in Figure 5 were carried out based on the numbers reported in [15] regarding the basic reproduction number of the dengue epidemic of 1990-91 in Brazil. The authors report that $R_{0}$ had an average value of 2.03 . They cite a previous work that took place in Mexico where the average reproduction number was found equal to 1.33 with a maximum of 2.41 [15]. The parameter values that we have chosen here are such that the basic reproduction number for both strains is about 2. However, which of the strains will be the winner depends on the initial conditions preexisting when the new strain arrived and on the lerel of susceptibility induced by the primary infection (Figures 4 and 5). Of course, if during the 
transient phase, a new wave of infected mosquitoes appears, it will not be possible to predict which strain will become endemic. In general, as Figure 3 shows, the region where both boundary equilibria are locally asymptotically stable is large and, according to our model, most dengue epidemics fall into this situation.

As shown in Figure 3, the existence of two locally asymptotically stable boundary equilibria as well as the existence of an (unstable)interior endemic equilibrium is guaranteed for a biologically feasible range of values of the superinfection indices $\sigma_{1}$ and $\sigma_{2}$. This range covers cases when primary infections induce resistance (decreased susceptibility) to secondary infections or when susceptibility is enhanced.

An interesting outcome of the model is that it seems that whenever $R_{i}>R_{j}$, then for feasible values of $\sigma_{i}$ (e.g. $\sigma_{i} \in(0.1,2)$ ), the interior equilibrium exists unless $\sigma_{j}$ is either very small or very large. In Figure 3 we illustrate the case when $R_{1}>R_{2}$ (see Figure 3 's legend for parameter values used). Therefore, we conclude that the presence of superinfection in our model forces the existence of the interior equilibrium. However, from our numerical studies, it appears that superinfection cannot induce stability in this state. Going back to Figure 3, the endemic interior equilibrium would cease to exists for a value of $\sigma_{2}=0.001$ but then only the boundary equilibrium $E_{1}^{*}$ would be asymptotically stable.

Gupta, Swinton and Anderson 10] show in a model for malaria that coexistence is a likely outcome wher cross-immunity is taken into account. Although malaria is a parasitic, not a viral disease, the mathematical structure of the model allows some comparisons with ours since both deal with a vector transmitted disease. Gupia et al. generalize directly the RossMacdonald model for malaria studied by Aron and May [1] introducing cross-immunity and two infected host subtypes: those that are infected and infectious, and those that are infected but uninfectious. Thus, essentially there is a reduction in the net number of infected individuals that can transmit the disease. However all infectec individuals can hold the parasite. In particular, the rate at which parasites become ineffective to transmission, i.e. the hosts becomes infected but not infectious, is exponential, guaranteeing the presence of positive densities however small) of each type of infected hosts for all time. Thus, the Gupta $\epsilon$ t al. model effectirely creates a refuge for each parasite strain. Moreorer, the total host population is considered constant. This factor alone when associated with cross-immunity :s enough to enhance coexistence in models for directly transmitted disease: 21], [16]. The assumption of constant host population size is achiered by $d \epsilon$. ining the 
recruitment rate in such a way as to balance the output from all system compartments.

In the case of our general modei all infected individuals are infectious; thus there are no refuges. Also, br definition, virulence is the extra death rate induced by the disease. This prevents the existence of a constant population size for the host. To define the recruitment rate for the total population so as to balance this outflow (and therefore achieve a constant size in the host population) would be equiralent to require that the extra-mortality rate is compensated exactly by the cure rate of the disease and that there is no net loss of individuals due to permanent immunity or death. Clearly a contradiction since, if that were the case, the more virulent a strain is, the higher its cure rate would be.

As pointed out before, we let the total host population vary constrained by the disease own dynamics and we do not provide refuges for the viral strains. If the population varies but this variation is independent of disease dynamics, the total host population size will be asymptotically constant with limiting asymptotic dynamics equivalent to constant population size models. However, even in this case (no virulence) our model predicts competitive exclusion of one of the strains.

The main reason that explains why in our model coexistence is an improbable outcome resides, we belie:e, in the coupling of two populations, each with a different pattern of disease progression.

The structure of the equations trat describe the transmission dynamics in the host population is that of an $S I R$ model with superinfection and variable population size. In a directl: transmitted disease with th:s structure and no virulence one would expect analogous results to those of lowak and May [21]: coexistence of both strairs as a rule since the total population in that case is asymptotically constant. However, our model also incorporates an $S I$ model without superinfection in the vector population. In a directly transmitted disease this structure would predict competitive exclusion of the strain with lower basic reproductive number [2].

When we couple both of these rypes of epidemics into one. our hostvector model (1-2), the outcome is competitive exclusion of one of the strains if at least one of the basic reproduc ive numbers is greater thar one. In a sense, the vector dynamics dominat The reason for this is that the recto-host relationship is asymmetric. The vector chooses the host. In this case we have modeled the contact rates according to a generalization of the Ross-Macdonald model: the contact rate is frequency dependent [7] (dejends on the ratios of vector numbers 
to host numbers for both types of strains). Thus, what our results show is that coexistence promoted by superinfection in the host population is 'broken' by frequency dependent dynamics in the biting (contact) rates, thus resulting in the competitive exclusion of one strain even when an interior steady-state exists.

Other models that incorporate cross-immunity and multiple strains have been studied [13], [14], [17]. However, we wish to compare our results with the original one that introduced this idea of competition of multiple strains in epidemic models, namely the Levin and Pimentel paper [13]. In summary, the conclusions of [13] are that in a rariable host population system coexistence is possible in a bounded region of parameter space. Outside this region, depending on the relative magnitudes of parameter values one of the two strains wins and competitively excludes the other. This model was originally designed for the theoretical study of mxomatosis as a control factor of an exponentially growing population. The fact that virulence is the growth regulatory factor in this model determines the existence of a coexistence region in parameter space. In the dengue model that we analyze here, the disease is not the unique factor that regulates growth although the model model is a generalization of Levin anc Pimentel's [13]. Besides, permanent immunity is explicitly introduced into the model. Even in the case when virulence is negligible competitive exclusion is the rule. The existence of frequency-dependent contact rates closes the window of coexistence.

The model analyzed here does not incorporates the effects of age structure. According to [26], dengue in tropical Asia affects particularly children with ages between 5 and 15 years old, with a modal age of 5 years. The same authors mention that in 1987 more than 600000 cases of dengue were reported in Southeast Asia with 24000 deaths: $90 \%$ of both cases and deaths were children. The risk of infection is obviously an age dependent factor. Moreover, the influence of physiological structure into the dynamics of dengue may have an influence in the likelihood of coexistence of both strains. The need for a model that incorporates age structure into the dengue population dynamics is thus justified.

Acknowledgements We wish to thank Car:os Castillo-Chavez for his continuous support and encouragement. This research was developed while JXVH was a visiting fellow of the Royal Soc:ety of London/Academia de la Investigación Cientifica, at the Zoology Depa:iment of the University of Oxford. JXVH acknowledges the support of CONACYT grant 400200-5-3551E. This research has been partially supported jo grant DEB-9253570 (Presi- 
dential Faculty Award) to Carlos Castillo-Chavez. This work was developed and finished while JXVH was a Postdoctoral Fellow at the Biometrics Unit of Cornell University. JXVH dedicates this work to the memory of Diego Bricio Hernández Castaños.

\section{References}

[1] Joan Aron and Robert M. May. The population dynamics of Malaria, pages 139-179. Population Dynamics of Infectious Diseases. Chapman and Hall, London, 1982.

[2] Hans J. Bremermann and Horst R. Thieme. A competitive exclusion principle for pathogen virulence. Journal of Mathematical Biology, 27:179-190, 1989.

[3] Vincenzo Capasso. Mathematical Structures of Epidemic Systems. Springer Verlag, New York, 1993. Lecture Notes in Biomathematics 97.

[4] Carlos Castillo-Chavez, Viggo Andreasen Herbert W. Hethcote, on A. Levin Si, and Wei-Min Liu. Epidemiological models with age structure, proportionate mixing, and cross-immunity. Journal of Mathematical Biology, 27:233-258, 1989.

[5] Carlos Castillo-Chavez, Wenzhang Huang, and Jia Li. Competitive exclusion in gonorreha models and other sexually-transmitted diseases. Technical Report 93-36, Mathematical Sciences Institute, Cornell University, 1993.

[6] Carlos Castillo-Chavez, Wenzhang Huang, and Jia Li. Dynamics of multiple pathogen strains in heterosexual epidemiological models. In Proceedings of the International Conference on Differential Equations Applied to Biology and Industry, number 00, pages 00-00, 1995.

[7] Carlos Castillo-Chavez, Jorge X. Velasco-Hernandez, and Samuel Fridman. Modelling contact structures in biology. Lecture Notes in Biomathematics, 100:454-492, 1995.

[8] Odo Diekmann, J.A.P. Heesterbeek, and J.A.J.Metz. On the definition and the computation of the basic reproduction ratio $r_{0}$ in models for 
infectious diseases in heterogeneous populations. Journal of Mathematical Biology, 28:365-382, 1990.

[9] D. J. Gubler. Dengue, volume 2 of The Arboviruses: Epidemiology and Ecology, pages 223-261. CRC Press, Inc., 1986.

[10] Sunetra Gupta, Johnatan Swinton, and Roy M. Anderson. Theoretical studies of the effects of heterogeneity in the parasite population on the transmission dynamics of malaria. Proceedings of the Royal Society of London B, 256:231-238, 1994.

[11] Scott B. Halstead. Dengue, pages 652-659. Tropical and Geographical Medicine. Mac Graw Hill, New York, 1984.

[12] Scott B. Healstead. The XXth century dengue pandemic: need for surveillance and research. World Health Statistics Quarterly, 45:292298, 1992.

[13] Simon Levin and David Pimentel. Selection of intermediate rates of increase in parasite-host systems. American Naturalist, 117(3):308$315,1981$.

[14] Marc Lipsitch and Martin A. Nowak. The evolution of virulence in sexually transmitted HIV/AIDS. Journal of Theoretical Biology, 174:427-440, 1995.

[15] C.A. Marques, O.P. Foratini, and E. Massad. The basic reproduction number for dengue fever in sao paulo state, brazil 1990-1991 epidemic. Transactions of the Royal Society of Tropical Medicine and Hygene, 88:58-59, 1994.

[16] Robert M. May and Martin N. Nowak. Superinfection, metapopulation dynamics, and the evolution of virulence. Journal of Theoretical Biology, 170:95-114, 1994.

[17] Jaime Mena-Lorca, Jorge X. Velasco-Hernandez, and Carlos CastilloChavez. Superinfection, virulence and density dependent mortality in an epidemic model. Technical Report BU 1299-M, Biometrics Unit, Cornell University, 1995.

[18] Helmar Nunes Moreira. Lienard-type equations and the epidemiology of malaria. Ecological Modelling, 60:139-150, 1992. 
[19] CNN World News. Deadly ferer sweeping Central, South Ameerica. Lucia Newman, Correspondant September 28, CNN Food and Health Page, http://www.cnn.com, 1995.

[20] Elizabeth A. C. Newton and Paul Reiter. A model of the transmission of Dengue fever with an evaluation of the impact of ultra-low volume (ULV) insecticide applications on Dengue epidemics. American Journal of Tropical Medicine and Hygene, 47:709-720, 1992.

[21] Martin N. Nowak and Robert M. May. Superinfection and the evolution of parasite virulence. Proceeedings of the Royal Society of London $B, 255: 81-89,1994$.

[22] World Health Organization. Selected health problems. World Health Statistics Quarterly, 44:208-233, 1991.

[23] World Health Organization. The urban crisis. World Health Statistics Quarterly, 44:189-197, 1991.

[24] Karamjit S. Rai. Aedes albopictus in the Americas. Annual Review of Entomology, 36:459-484, 1991.

[25] William C. Reeves, James L. Hardy, William K. Reisen, and Marilyn M. Milby. Potential effect of global warming on mosquito-borne arboviruses. Journal of Medical Entomology, 31:323-332: 1994.

[26] Donald S. Shepard and Scott B. Halstead. Dengue (with notes on Yellow fever and Japanese encephalitis), pages 303-320. Disease Control Priorities in Developing Countries. Oxford University Press, 1993.

[27] David Tilman. Competition and biodiversity in spatially structured habitats. Ecology, 75(1):2-16, 1994.

[28] Jorge X. Velasco-Hernandez. A model for Chagas disease involving transmission by vectors and blood transfusion. Theoretical Population Biology, 46(1):1-31. 1994. 
Figure captions

Table 1. Dengue hemorrhagic fever cases and deaths reported to the World Health Organization regional offices. Data obtained from Halstead (1992).

Table 2. Parameter definitions and values used in the simulations illuestrated in the figures.

Figure 1. Graph on the parameter space $\left(\sigma_{1}, \sigma_{2}\right)$ for case 1 of Lemma 1 . In this case $R_{1}<R_{2}, f(0)=0$. The shaded area corresponds to parameters values that render the boundary equilibrium for strain 1 locally asymptotically stable.

Figure 2. Graph on the parameter space $\left(\sigma_{1}, \sigma_{2}\right)$ for case 2 of Lemma 1 . In this case $R_{1}<R_{2}, f(0)=0$. The shaded area corresponds to parameters values that render the boundary equilibrium for strain 1 locally asymptotically: stable.

Figure 3. Region of parameter space $\left(\sigma_{1}, \sigma_{2}\right)$ where both boundary equilibria are locally asymptotically stable. Fixed parameter values are $r=0.71 /$ day, $\mu=0.000039 /$ day, $\delta=0.71 /$ day $, h=0.9775, \alpha_{1}=0.002, \alpha_{2}=0.015, \beta_{1}=0.001, \beta_{2}=0.001, c=10$, $\widehat{T}=50000, \widehat{N}=25000$. The corresponding basic reproduction numbers are $R_{1}=2.4$ and $R_{2}=2.08$.

Figure 4. Phase plot in the space $\left(I_{1}, I_{2}\right)$ for values of the superinfection indices outside the shaded area shown in Figure 3. The graph was computed with the same parameter values shown in Figure 3 but with $\sigma_{1}=5, \sigma_{2}=0.05$, and positive disease-induced death rates $e_{1}=0.0001 /$ day and $e_{2}=0.0005 /$ day. These parameter values give $\sigma_{2}<g^{-1}\left(\sigma_{1}\right)=0.1$. In this case strain 1 competitively excludes strain 2 . Note that the final outcome of the disease (which strains wins) is independent of initial conditions.

Figure 5. Phase plot in the space $\left(I_{1}, I_{2}\right)$ for values of the superinfection indices outside the shaded area shown in Figure 3. The graph was computed with the same parameter values shown in Figure 3 but with $\sigma_{1}=1, \sigma_{2}=4.2$, and positive disease-induced death rates $e_{1}=0.0001 / d a y$ and $e_{2}=0.0005 /$ day. The presence of a saddle point in the interior of the region and the existence of a separatrix may be conjectured. Note that the final outcome of the disease (which strains wins) depends on initial conditions. 
Figure 6. Time plot of model (1-2) for a period of 5 years. The graph shows the total numbers of infected individuals for each strain $I_{1}+Y_{1}$ and $I_{2}+Y_{2}$. Parameter values are the same as for Figure 3 except for the following: $\alpha_{1}=0.005, \alpha_{2}=0.005, \beta_{1}=0.005$, $\beta_{2}=0.007, \sigma_{1}=4, \sigma_{2}=1.2$. There are two curves, one for each strain. For about 3 years both strains seem to increase and coexist. Only in the fourth year strain 1 clearly wins over strain 2 . Note that strain 2 increases faster at the beginning of the epidemic but it is this strain the one that goes extinct. 


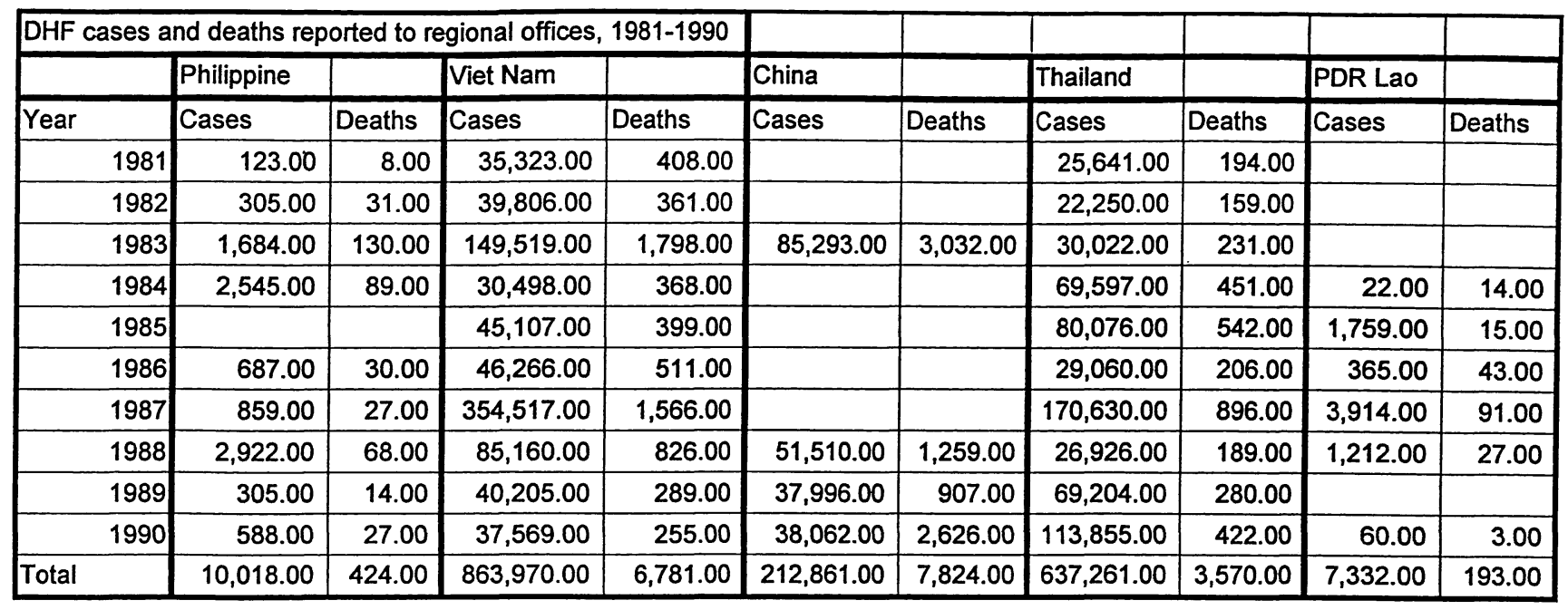




\begin{tabular}{|l|l|l|}
\hline Symbol & Parameter definition & value \\
\hline$h$ & host recruitment rate & variable \\
\hline$u^{-1}$ & host life expectancy & 70 years \\
\hline$r^{-1}$ & mean length of infectious period in host & 14 days \\
\hline$\alpha_{i}$ & vector per capita infection rate (biting rate $\times$ vector infection probability) & $(0,0.05)$ \\
\hline$\beta_{i}$ & host per capita infection rate (biting rate $\times$ host infection probability) & $(0,0.05)$ \\
\hline$q$ & vector recruitment rate & variable \\
\hline$\delta^{-1}$ & vector life expectancy & 14 days \\
\hline$c$ & rescaling parameter $\left(\alpha_{i} / c\right.$ and $\beta_{i} / c$ infection rates when $N$ small $)$ & 1 \\
\hline$\omega_{i}$ & saturation parameter $\left(\alpha_{i} / \omega_{i}\right.$ and $\beta_{i} / \omega_{i}$ give maximum infection rates $)$ & 0.5 \\
\hline$e_{i}$ & disease-induced per-capita death rate & variable \\
\hline$\sigma_{i}$ & susceptibility index to $\operatorname{strain} i$ & $(0,5)$ \\
\hline
\end{tabular}




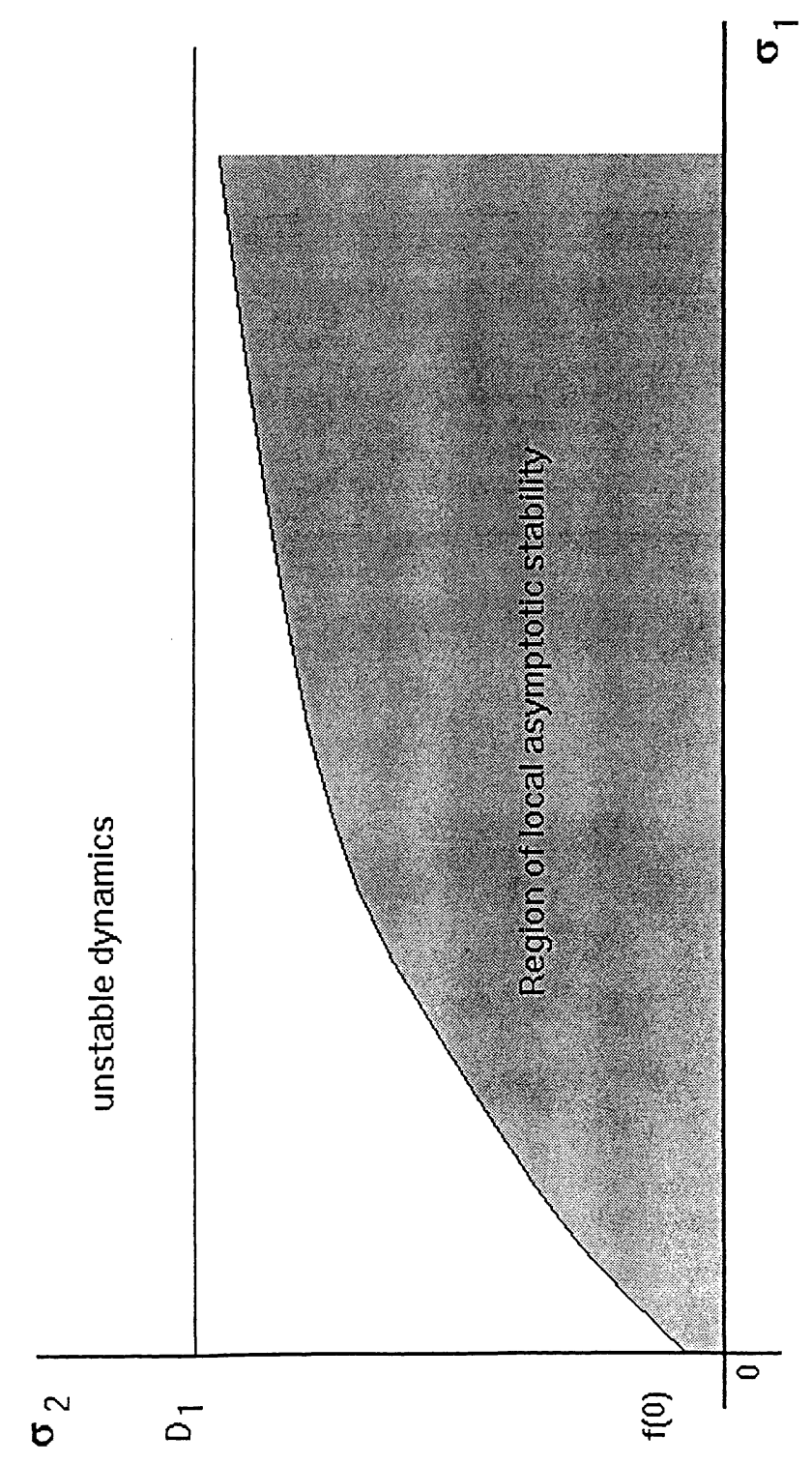




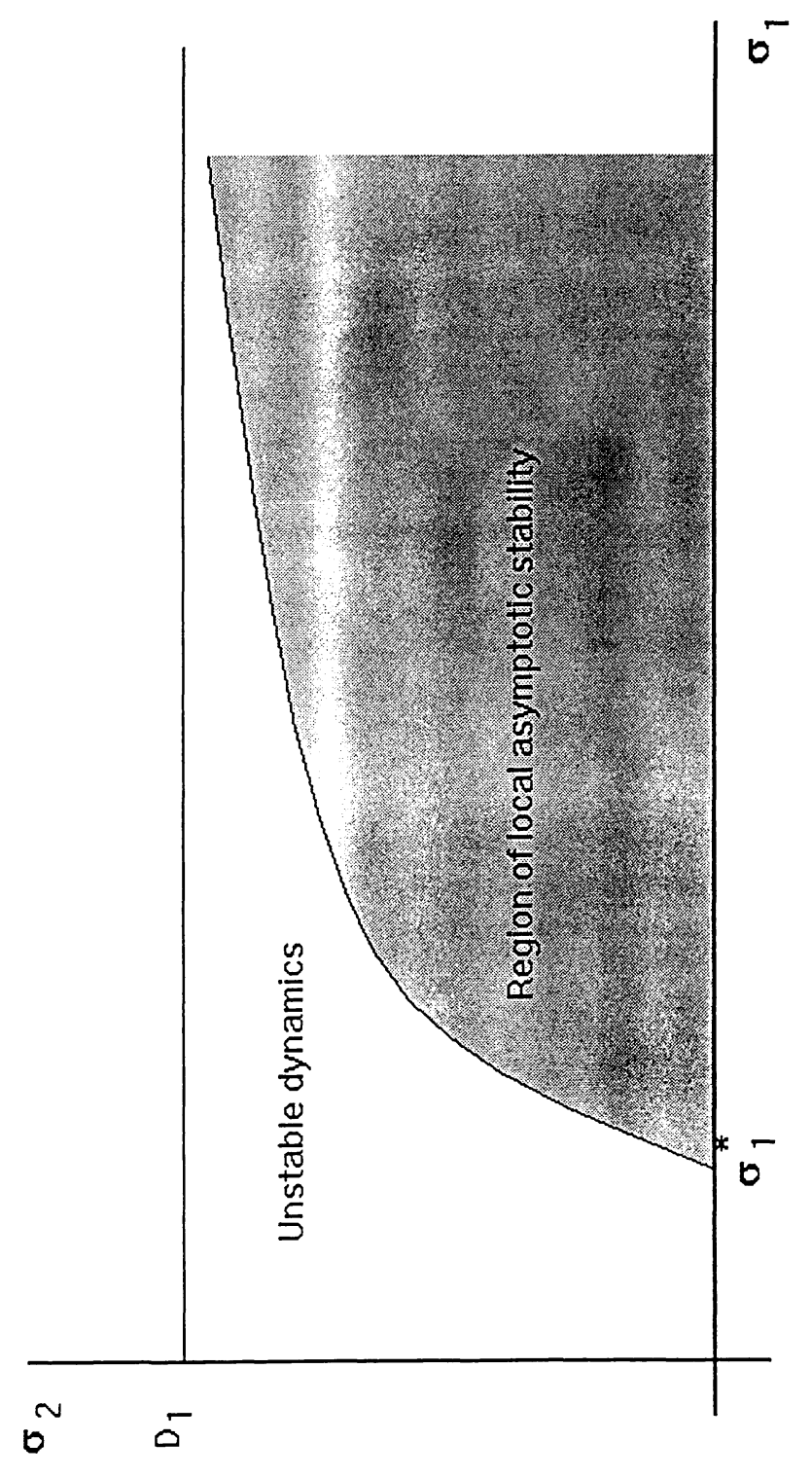




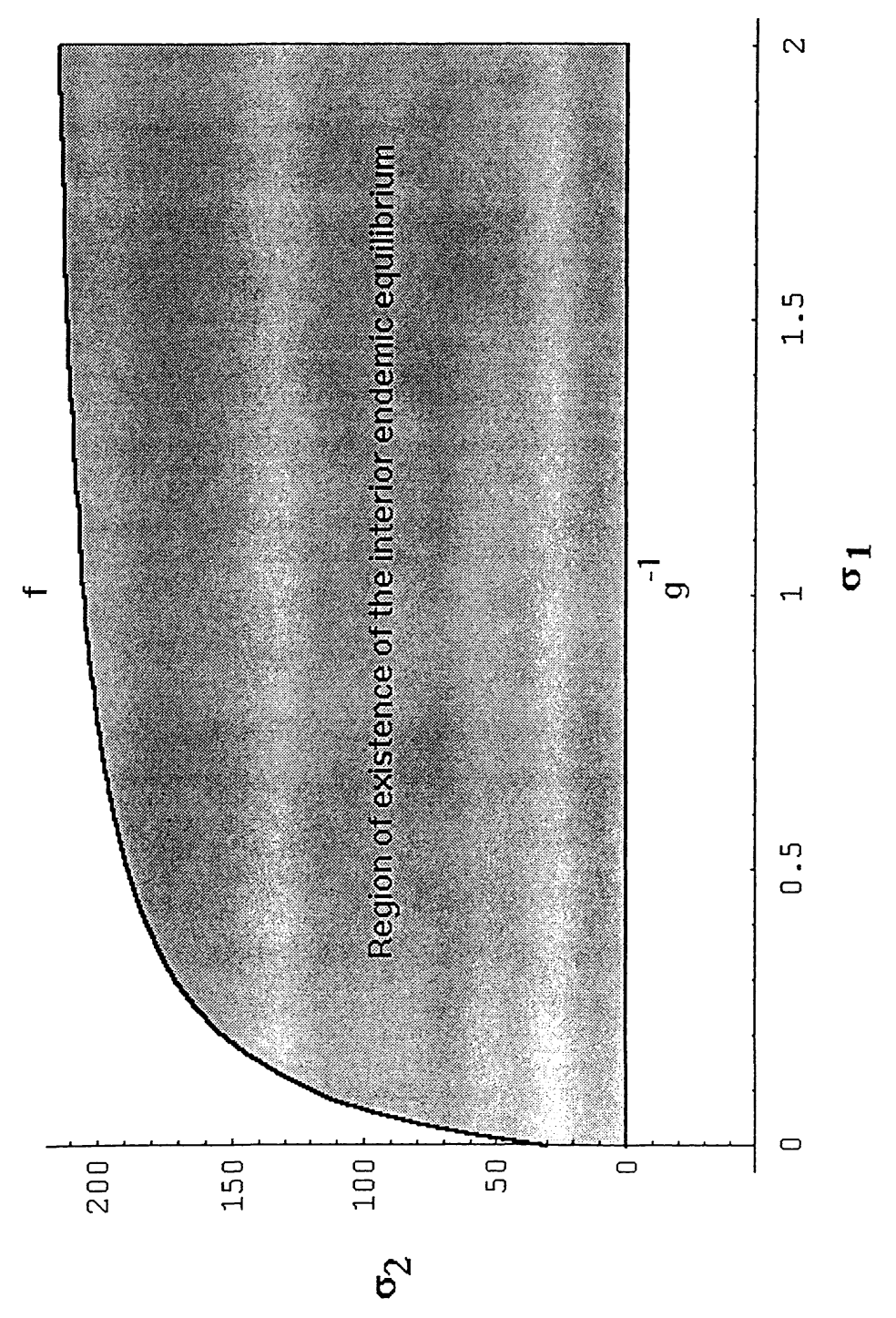




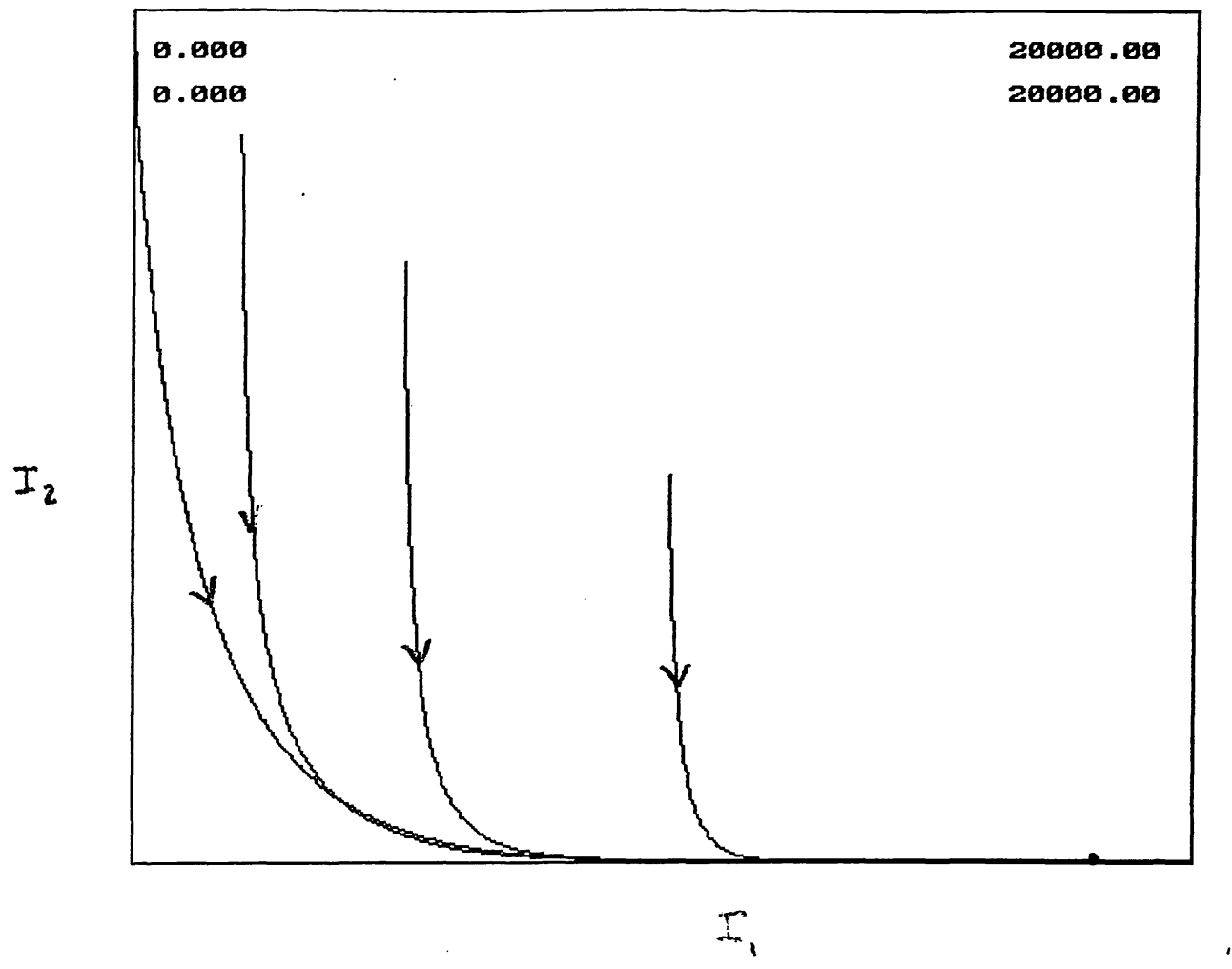

4 


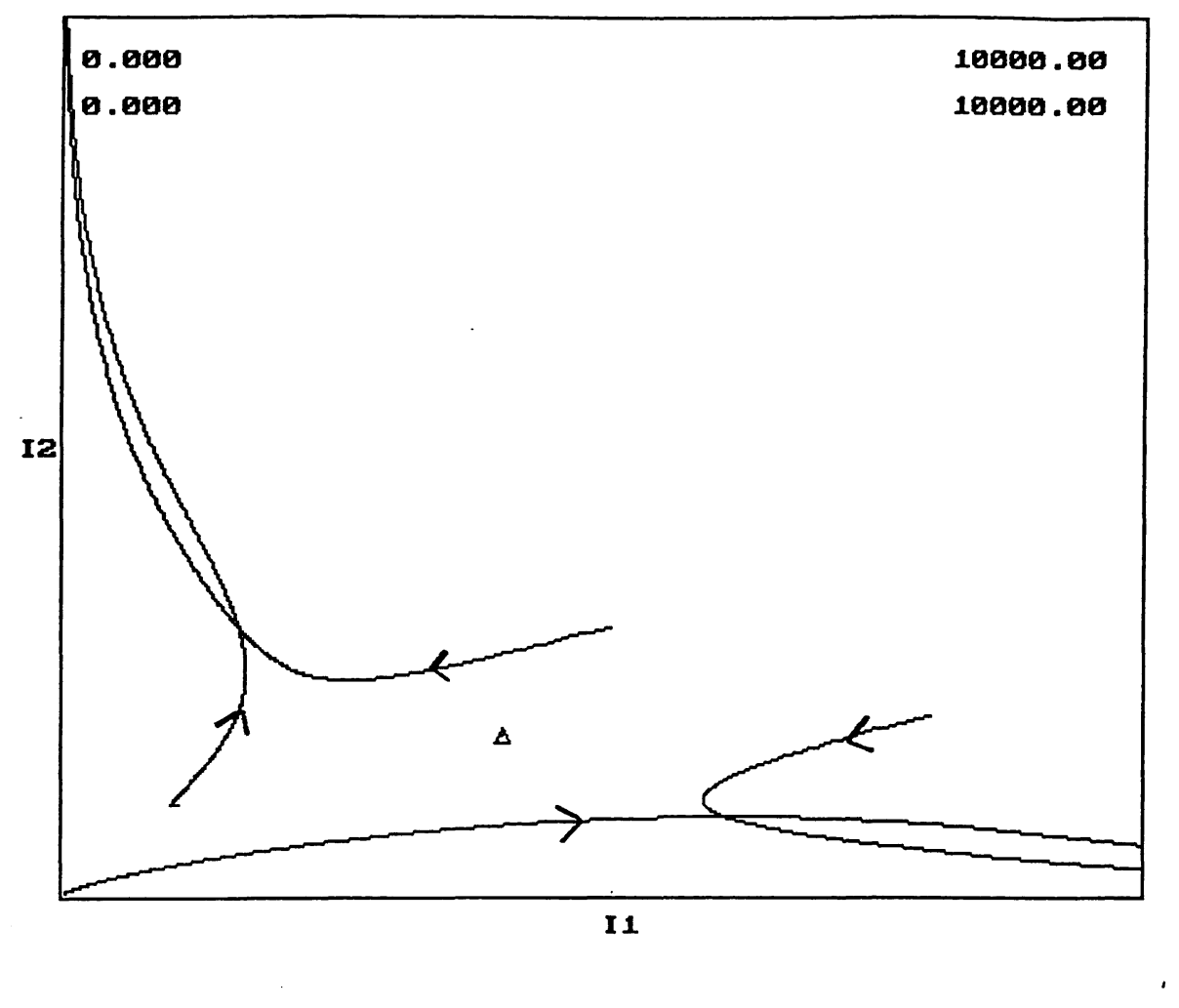

$\exists \quad \therefore \quad$ 


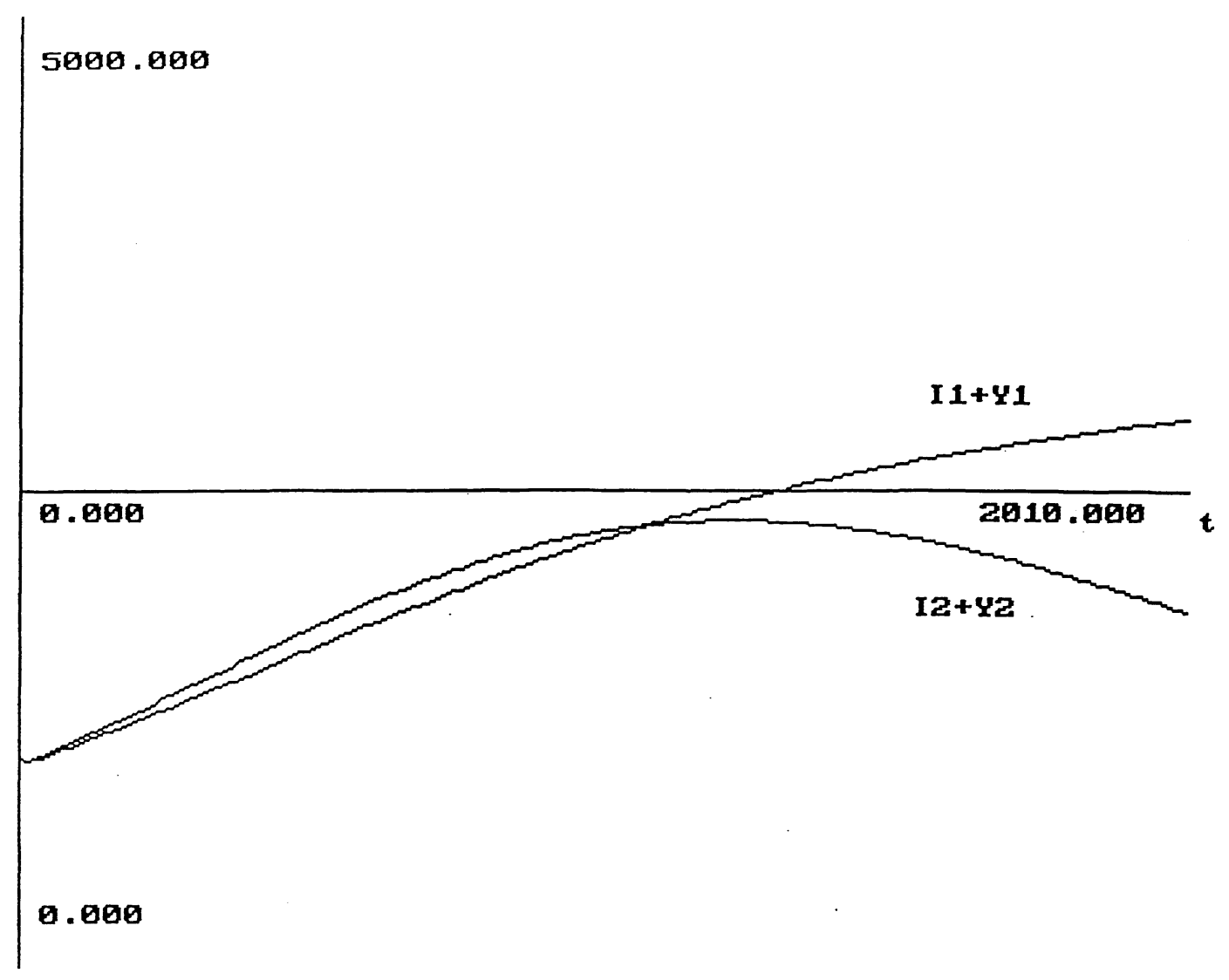

